\title{
Two Classes of Improved Quickest Spectrum Sensing Approaches in Cognitive Radio
}

\author{
Qi Zhao ${ }^{1} \cdot$ Bingqian Wang ${ }^{1} \cdot$ Xiaochun $\mathrm{Li}^{1}$
}

Published online: 12 July 2016

(C) The Author(s) 2016. This article is published with open access at Springerlink.com

\begin{abstract}
In this work, we mainly discuss two classes of quickest spectrum sensing approaches. The first is the improved single-channel detection based on cumulative sum (CUSUM) and generalized likelihood ratio, applied with primary user's signal unknown. The other is wideband spectrum sensing based on Gobar transform and Wigner-Ville transform, implementing the transformation of the parallel sensing in time-frequency domain from the serial sensing in time-domain. The quickest spectrum sensing framework is developed to investigate the detection delay of the two approaches. Performance is evaluated using theoretical analysis and numerical simulations. Simulation results show that the improved single-channel detection is close to the CUSUM in performance though with unknown parameters. Moreover, in the wideband spectrum sensing, the detection delay of the ameliorated method is obviously superior to that of STFT with the certain false alarm interval.
\end{abstract}

Keywords Cognitive radio - Spectrum sensing - Quickest detection · CUSUM - GLR

\section{Introduction}

In recent years, cognitive radio has attracted intensive research because of pressing demand of efficient frequency spectrum usage. The basic idea is to allow secondary users to use the spectrum when primary users are not transmitting. A key problem in cognitive radio is that

\footnotetext{
Qi Zhao

zhaoqi@buaa.edu.cn
}

Bingqian Wang

1165335368@qq.com

Xiaochun Li

lixc680@126.com

1 School of Electronic Information Engineering, Beihang University, Beijing 100191, China 
the secondary users need to quit the frequency band as quickly as possible if the corresponding primary radio emerges. Essentially, spectrum sensing is used to detect the change of the spectrum activities. For spectrum detection algorithm, there are two typical frameworks, namely block detection and quickest detection. The block detection is based on blocks of observations, and the decision method of the block detection is made at the end of each block. Quickest detection is a sequential change-point detection approach. Meanwhile, there are two major drawbacks of the traditional block detection. One is that the decision is unreliable for a small block size and considerable detection delay occurs for a large block size. The other is that the observations both before and after a change may appear in the same block, thus the decision may be invalid (the block detection is a binary hypothesis test that the primary user exists or not). Some classical detection features, such as energy detection, matched filtering detection and cyclic-feature detection, whose frameworks are block-based, are used to obtain the minimal miss probability subject to given false alarm constraints. Compared with them, quickest detection is used to obtain the minimal detection delay subject to given false alarm constraints, which is more efficient for the dynamic spectrum access.

Quickest detection performs a statistical test to detect the change of distribution in observations in order to attain an agile and robust spectrum sensing [1]. If a primary user stops transmission, then a secondary user should detect this event quickly, in order to be able to start its own transmission. A small detection delay will allow secondary users to take more transmission opportunities. On the other hand, if the primary user starts transmission, the secondary user should detect this event as quickly as possible, in order to vacate the band for the primary user. A small detection delay will allow the design of a spectrum reuse scheme that has minimal impact on the licensed users. Of course, the desire to reduce the detection delay should be balanced with a certain false alarm constraint. Our goal is to establish a statistical framework to analyze detection delay, subject to certain false alarm constraints, and more importantly to design a scheme that can minimize the delay.

The traditional methods for the quickest detection are the well-known cumulative sum (CUSUM) detection and generalized likelihood ratio (GLR) detection. CUSUM algorithm, simple and fast, is sensitive to uncertainty in parameters, as discussed in several papers, e.g. [2]. The GLR algorithm is used for the primary user with unknown parameters, and can get an estimate of the full samples. However, it is unable to create iteration, resulting in long time running and less application in practice. Therefore we adopt the short detection time of CUSUM algorithm and unknown parameters estimation of GLR algorithm to propose a new method, applied to the quickest detection for unknown primary users. Later theoretical analysis and numerical simulations are performed. Besides, we set foot in the wideband spectrum sensing. The traditional quickest detection is performed well in the time domain for the single-channel. In this paper, we have introduced the quickest detection to the multi-channel. However, in the multi-channel, if only perform the quickest detection in the time domain, we cannot distinguish different frequency. Fortunately, Short-time Fourier transform (STFT), Gabor transform, or Wigner-Ville transform is utilized to distinguish different channels and transform the time- detection to the frequencydetection. The method realizes the multi-channel parallel detection. Compared to the single-channel serial spectrum sensing, the spectrum efficiency of multi-channel parallel sensing could be improved. Then we perform comparisons between STFT and the improved methods.

The rest of the paper is organized as follows. In Section II, we introduce the model. In Section III and IV, two improved quickest spectrum sensing approaches are respectively 
described in details and related mathematic formulas are derived. In Section V, includes extensive simulation results and analyses. In Section VI, we offer some conclusions.

\section{System Model}

The quickest detection is a dynamic process, namely allowing the secondary user access the band when the primary users disappear. In this process, we can use modeling methods to express clearly, (1) and (2) describes two modeling methods of quickest detection. $s(i)$ is the primary signal and $u(i)$ is additive white Gaussian noise(AWGN) with mean zero and variance $\sigma^{2} . s(i)$ is also considered to be white and Gaussian with mean zero and variance $P$, which is a reasonable assumption [3]. When the primary user appears at an unknown time $\tau, y(i)$ becomes:

$$
y(i)= \begin{cases}u(i) & i=1,2, \ldots, \tau-1 \\ s(i)+u(i) & i=\tau, \tau+1, \ldots\end{cases}
$$

where $s(i) \sim N(0, P), u(i) \sim N\left(0, \sigma^{2}\right)$, before $\tau$, the samples obey the distribution of $P_{0}$ (namely mean zero and variance $\sigma^{2}$ ), while after that, the samples obey the distribution of $P_{1}$ (namely mean zero and variance $P+\sigma^{2}$ ) y(i) also becomes:

$$
y(i)= \begin{cases}u(i) & i=1,2, \ldots, \tau-1 \\ A \sin \left(2 \pi i f_{c} t_{s}+\theta\right)+u(i) & i=\tau, \tau+1, \ldots\end{cases}
$$

where $A$ is the amplitude of the primary radio signal, which is confined within an interval [ $\left.A_{\max }, A_{\min }\right]$ (when $A<A_{\min }$, the impact of the secondary radio signal on the primary radio is negligible), $f_{c}$ is the carrier frequency, $f_{\mathrm{s}}$ is the sampling frequency, $f_{s}=1 / t_{s}, \theta$ is the phase of the primary user's signal. It can be modeled as a quickest detection problem for the sequence of distribution change. In the detection, we accumulate the processed sample points and judge each sample point. And once we determine the primary user signal "appear" or "exit" band, the secondary user immediately responses to current band occupancy.

Assume that $t$ is the detection time when the primary user appears. Following [4], we use two performance evaluation indexes, i.e. the worst detection delay $\overline{T_{1}}$ and the mean time to false alarms $\overline{T_{0}}$ :

$$
\begin{gathered}
\bar{T}_{1}=\sup _{\tau \geq 1} \operatorname{ess} \sup E_{p_{1}}\left\{T=t-\tau \mid t \geq \tau, y_{1}^{\tau}\right\} \\
\bar{T}_{0}=E_{p_{0}}\{t\}
\end{gathered}
$$

where $T=t-\tau$ is defined as the detection delay. When $t \geq \tau, y_{1}^{\tau}$ is the observation sequence before the primary user appears, and $E_{p_{1}}$ denotes the conditional expectation under the assumption that the primary user appears at $\tau$. Otherwise, $\{t<\tau\}$ is a false alarm event. The quickest detection problem for cognitive radio is formulated as to obtain the minimum worst detection delay subjected to given false alarm constrains. Obviously, a small $\bar{T}_{1}$ and a large $\bar{T}_{0}$ are required. In the traditional CUSUM algorithm, $\overline{T_{1}}$ and $\overline{T_{0}}$ can be approximated as:

$$
\bar{T}_{1}=\frac{\lambda}{E_{p_{1}}\left[l_{i}^{P}\right]}
$$




$$
\bar{T}_{0}=e^{\lambda}
$$

where $\lambda$ is a detection threshold. $E_{p_{1}}\left[l_{i}^{P}\right]=D\left(p_{1} \| p_{0}\right)$, where $D(\cdot \| \cdot) \psi$ denotes KullbackLeibler divergence.

The single-channel system model can be extended to wideband signal model $[5,6]$. The received signal in the time domain can be represented as:

$$
y(i)= \begin{cases}u(i) & i=1,2, \ldots, \tau-1 \\ s_{k}(i)+u(i) & i=\tau, \tau+1, \ldots\end{cases}
$$

where $k$ shows the different primary users, and $i$ is the time of different primary users occur, which can be different from each other. It is difficult to detect the change-point of different primary users according to the observation sequence in time domain. Therefore, we come up a method that the cognitive user differentiates the different primary users in frequency domain and obtain the detection time in time domain.

\section{The Improved Quickest Spectrum Sensing Approach with Unknown Parameters in a Single Channel}

\subsection{Algorithm}

The CUSUM algorithm is based on the perfect knowledge of the distributions [3]. However, in many situations, the distribution parameters of primary users are usually unknown (e.g. the amplitude and phase) due to channel fading and noise interference. GLR algorithm, proposed by Lorden [7], implements the maximum likelihood ratio estimate. Unfortunately, there is no recursive expression for the GLR computation. So all observations need to be stored and the metrics need to be recomputed in all time slots. This makes the GLR algorithm infeasible for practical system implementation. Therefore we consider combining CUSUM algorithm and the GLR algorithm to improve the traditional quickest detection algorithm. In the improved method, GLR algorithm is applied to narrow the range of parameters. Because of the influence of channel fading and other unknown factors, it is difficult to clear the variance of primary user signal, but we can know $P \in\left[P_{\text {min }}, P_{\text {max }}\right]$. We carry on two times of parallel CUSUM detection of $P_{\text {max }}$ and $P_{\text {min }}$ in threshold $\lambda_{1}$ and $\lambda_{2}$ respectively to obtain the detection time. Every CUSUM detection method is described as below:Firstly, to obtain the logarithmic likelihood ratio:

$$
l_{k}(t)=\log \left(\frac{p_{1 k}\left(y_{t}\right)}{p_{0 k}\left(y_{t}\right)}\right)
$$

where, $k$ is the detection times. Then, to obtain the cumulative sum:

$$
s_{k}(t)=\max \left(s_{k}(t-1)+l_{k}(t), 0\right)
$$

the detection time is

$$
t_{k}=\inf \left(t \mid s_{k}(t) \geq \lambda\right)
$$

the final detection time is

$$
T=\inf \left(t_{k}, k=1,2 \ldots, n\right)
$$


Then with $P_{\max }$ and $P_{\min }$, we can get two times of detection time $t_{1}$ and $t_{2}$. From [8], we could basically think primary user signals appear in between $\left[t_{1}, t_{2}\right]$, by using the GLR parameter estimation in between $\left[t_{1}, t_{2}\right]$ :

$$
P^{*}= \begin{cases}P_{\max }, & t-v \leq \frac{2 \hat{y}}{P_{\max }+\sigma^{2}} \\ \frac{2 \hat{y}}{t-v}-\sigma^{2}, & \frac{2 \hat{y}}{P_{\max }+\sigma^{2}} \leq(t-v) \leq \frac{2 \hat{y}}{P_{\min }+\sigma^{2}} \\ P_{\min }, & (t-v) \geq \frac{2 \hat{y}}{P_{\min }+\sigma^{2}}\end{cases}
$$

where $\hat{y}=\sum_{i=v+1}^{t} y_{i}^{2}, P^{*}$ is the power that maximizes $f(P)=\frac{P}{2\left(P+\delta^{2}\right) \delta^{2}} \widehat{y}+(t-v) \frac{1}{2} \ln \left(\frac{\sigma^{2}}{P+\sigma^{2}}\right)$ over the region $P_{\min } \leq P \leq P_{\max }$. Finally, get the final detection $t$ in the threshold $\lambda_{3}$ with the estimated $P^{*}$ through the CUSUM detection.

\subsection{Asymptotic Performance Analyses}

We will analyze the asymptotic performance of $\overline{T_{1}}$ and $\overline{T_{0}}$ as expressed in (3) and (4), separately. For simplicity, we also assume $k=3$, namely the detection procedure contains three stages: initial detection, parameter refinement and final detection.

First, we need to study the impact of the mismatched parameter $P$ on the CUSUM detection. Suppose that an imperfect $\widehat{P}$ is used as the true value in the CUSUM detection. It is easy to verify that, by applying the Brownian motion approximation, $\bar{T}_{1}$ is approximated by

$$
\bar{T}_{1} \approx \frac{\lambda}{E_{p_{1}}\left[\widehat{l_{i}^{P}}\right]}
$$

where

$$
\begin{aligned}
E_{p_{1}}\left[l_{i}^{P}\right] & =E_{p_{1}}\left[\log \left(\frac{p_{1}^{P}\left(y_{i}\right)}{p_{0}\left(y_{i}\right)}\right)\right] \\
& =E_{p_{1}}\left[\log \left(\frac{p_{1}^{P}\left(y_{i}\right)}{p_{0}\left(y_{i}\right)}\right)\right]-E_{p_{1}}\left[\log \left(\frac{p_{1}^{P}\left(y_{i}\right)}{p_{1}^{P}\left(y_{i}\right)}\right)\right] \\
& =D\left(p_{1}^{P} \| p_{0}\right)-D\left(p_{1}^{P} \| p_{1}^{\widehat{P}}\right)
\end{aligned}
$$

By applying the Information Inequality, which states

$$
D\left(p_{1}^{P} \| p_{1}^{P}\right) \geq 0
$$

We have

$$
E_{p_{1}}\left[\widehat{l} l_{i}^{P}\right] \leq E_{p_{1}}\left[l_{i}^{P}\right]
$$

From [8] we know, under the not perfect parameter $\hat{P}$, the first detection time is: 


$$
i=\arg \min _{j}\left(D\left(p_{1}^{P} \| p_{1}^{p_{j}}\right)\right)
$$

So we can be defined the parameter in a test as:

$$
P_{i}^{*}=\arg \min _{\hat{P} \in \mathrm{P}_{i}}\left(D\left(p_{1}^{P} \| p_{1}^{\hat{P}}\right)\right)
$$

where $P_{i}^{*}$ is the collection of the estimate parameter of the primary user in the two parallel CUSUM detection of the $i$ time. From [8], we can design

$$
I=D\left(p_{1}^{P} \| p_{0}\right)
$$

Therefore, for the detection of the above process, the average run length of the worst detection delay can be expressed as:

$$
\bar{T}_{1} \approx \frac{\lambda_{1}}{I-D\left(p_{1}^{P} \| p_{1}^{P_{1}^{*}}\right)}+\frac{\lambda_{2}}{I-D\left(p_{1}^{P} \| p_{1}^{P_{1}^{*}}\right)}+\frac{\lambda_{3}}{I-D\left(p_{1}^{P} \| p_{1}^{P_{2}^{*}}\right)}
$$

Due to the maximum likelihood ratio criterion, when $\lambda_{1}$ and $\lambda_{2}$ is large, $D\left(p_{1}^{p} \| p_{1}^{p_{2}^{*}}\right)$ convergence to 0 . Besides, as the order number of $\lambda_{3}$ is higher than $\lambda_{1}$ and $\lambda_{2}$, it can be approximated as

$$
\bar{T}_{1} \approx \frac{\lambda_{3}}{I}=\frac{\lambda_{3}}{D\left(p_{1}^{P} \| p_{0}\right)}
$$

Through the above analysis we can know, when $\lambda_{1}, \lambda_{2}$ and $\lambda_{3}$ meet the preset conditions, for the same threshold, the average run length of worst detection delay of the improved method is approximate to the CUSUM algorithm. The basis of the average run length of false alarm intervals is false alarm events. In the improved method, the main factors affecting the false alarm is the valuation of $P$. Similar to the derive of $\overline{T_{1}}, \overline{T_{0}}$ mainly depends on the stage detection of the $\lambda_{3}$. Therefore, according to the GLR algorithm, we can conclude the $\overline{T_{0}}$ in the improved method is

$$
\bar{T}_{0} \geq 1 / a
$$

where $a$ is design parameter. Threshold $\lambda$ is

$$
\lambda=-\ln \{a / b\}
$$

where $\quad b=3 \ln \left\{a^{-1}\left(1+\frac{1}{D\left(p_{1, P \min } \| p_{0}\right)}\right)^{2}\right\}$.

\section{Two Novel Quickest Spectrum Sensing Approaches for Wideband Cognitive Radio Respectively with Gabor and Wigner-Ville Transform}

\subsection{Algorithm}

Gabor transform is the optimal windowed Fourier transform. Its significance lies in that the Gabor transform brings time-frequency analysis in real sense. The Gabor transformation can achieve frequency localization, which can not only provide all the information on the 
whole but also provide more vary information at any local time. In short, it can provide the localization information in time and frequency at the same time Gabor transform is described as below:

$$
G_{f}(a, b, \omega)=\int_{-\infty}^{\infty} f(t) g_{a}^{*}(t-b) e^{-i \omega t} d t
$$

where $f(t)$ is the signal, and $g_{a}(t-b)$ is a window function of time localization. Among them, the parameters $b$ is used for parallel moving window, in order to cover the whole time domain. If we integral the parameters $b$, we can conclude:

$$
\int_{-\infty}^{\infty} G_{f}(a, b, \omega) d b=\hat{f}(\omega), \omega \in R
$$

where $\hat{f}(\omega)$ is the Fourier transform of $f(t)$. Namely, when the moving distance of the window from minimum to maximum, Gobar becomes the Fourier trans-form. Through theoretical derivation, it can be drawn that the product of the window width and height is a fixed value under the Gauss window. As it is described below:

$$
[b-\sqrt{a}, \quad b+\sqrt{a}] \times\left[\omega-\frac{1}{a \sqrt{a}}, \quad \omega-\frac{1}{a \sqrt{a}}\right]=2
$$

Rectangular time-frequency window: the width is $2 \sqrt{a}$, the height is $1 / \sqrt{a}$.

Wigner-Ville transform is described as below [9]:

$$
W_{x}(t, f)=\int_{-\infty}^{+\infty} x\left(t+\frac{\tau}{2}\right) x\left(t-\frac{\tau}{2}\right) e^{-j 2 \pi f \tau} d \tau
$$

where $x(t)$ is the signal, but in practical numerical implementation, we must have the Wigner-Ville dispersion [10]. The discrete expression defined for Wigner-Ville is

$$
W_{x}(n, k)=2 \sum_{m=-(N-1) / 2}^{(N-1) / 2} x(n+m) x^{*}(n-m) e^{-j 4 \pi m k / N}
$$

where $f=\frac{f_{s} k}{2 N}, t=\frac{n}{f_{s}}, \tau=\frac{m}{f_{s}}$. Gabor transform and Wigner-Ville transform are both same as STFT, which are all performed Fourier transform after some mathematical processing. So we can carry on the same modeling and CUSUM detection process for the processed data. With the translation, the one-dimensional time domain signal $y(i)$ will become a twodimensional matrix $Y_{m, n}$, in which $m$ is the time sampling-point and $n$ is the frequency sampling-point. But the complex $Y_{m, n}$ not as a sample sequence of spectrum sensing, this is because of the fast detection algorithm of statistics need to be compared with each other, and compare with the preset threshold, so the need for $Y_{m, n}$ to do some conversion. A transformational observation sequence is applied for quickest detection [11, 12]:

$$
Y_{m, n}=\operatorname{Re}\left[Y_{m, n}\right]+\operatorname{Im}\left[Y_{m, n}\right]
$$

For the sampling-point $Y_{m, n}$, the log-likelihood ratio at $\forall m$ and $\forall n$ can be defined as:

$$
l_{m, n}=\log \left(\frac{f_{1 n}\left(\bar{X}_{m, n}\right)}{f_{0 n}\left(\bar{X}_{m, n}\right)}\right) \quad m=1,2,3 \ldots, \quad n=1,2,3 \ldots,
$$


where $f_{1 n}$ and $f_{0 n}$ represent the probability density of the sampling-value in the environment of $H_{1}$ and $H_{0}$ after windowing, respectively. then, to obtain the cumulative sum

$$
s_{m, n}=\max \left(s_{m-1, n}+l_{m, n}, 0\right)
$$

the detection time is

$$
t_{n}=\min \left\{m \mid s_{m, n} \geq \gamma\right\}
$$

\subsection{Asymptotic Performance Analyses}

The random variables $y_{i} \sim N\left(\mu_{i}, \sigma_{i}^{2}\right), i=1,2, \ldots, n$ and they are independent of each other. The linear combination of them is: $C_{1} x_{1}+C_{2} x_{2}+\cdots+C_{n} x_{n}\left(C_{1}, C_{2}, \ldots C_{n}\right.$ are constants not all for 0 ). Still obey the Gauss distribution, namely:

$$
C_{1} x_{1}+C_{2} x_{2}+\cdots+C_{n} x_{n} \sim N\left(\sum_{i=1}^{n} C_{i} \mu_{i}, \sum_{i=1}^{n} C_{i} \sigma_{i}^{2}\right)
$$

Definition: a finite sequence set to a length of, is defined as the point of discrete Fourier transform

$$
Y(k)=D F T[y(n)]=\sum_{i=0}^{N-1} y(n) e^{-j \frac{2 \pi}{N} k n}, \quad k=0,1, \ldots, N-1
$$

where $N>M$.

This method uses the Gauss window segmentation signal in time domain, the window length is $M=2 \sqrt{a}$, and the length of FFT is also $M$. Based on the above discussion, we can make the following inference. Before Gabor transform, the distribution of primary user signal with length $N$ [13]:

$$
s_{k}(i) \sim N\left(0, P_{k}\right), \quad i=1,2,3, \ldots N
$$

where $P_{k}$ is the variance of primary user signal, $k$ is the number of the primary users. The carrier frequency of each primary user signal is $f_{c, k}=\left(f_{s} / N\right) * l$ and $l$ is random integer. We can think that after the primary user signals are sampled, the side lobes are infinitesimal, i.e. only at a certain frequency, the amplitude is not zero, and the influence between the primary users is infinitely small. So after Gabor transform, if only noise exist $Y_{m, n}$ is described as below:

$$
Y_{m, n} \sim N\left(0, M \sigma^{2}\right), \quad m=1,2,3, \ldots \frac{N}{M}, \quad n=1,2,3, \ldots, M
$$

if the primary user is in the present frequency band, $Y_{m, n}$ is:

$$
Y_{m, n} \sim N\left(0, M P_{k}+M \sigma^{2}\right), \quad m=1,2,3, \ldots \frac{N}{M}, \quad n=1,2,3, \ldots, M
$$

According to [11], if only noise exits,

$$
\operatorname{Re}\left[Y_{m, n}\right] \sim N\left(0, \frac{M \sigma^{2}}{2}\right), \quad \operatorname{Im}\left[Y_{m, n}\right] \sim N\left(0, \frac{M \sigma^{2}}{2}\right)
$$

If the primary user and noise exit at the same time, 


$$
\operatorname{Re}\left[Y_{m, n}\right] \sim N\left(0, \frac{M \sigma^{2}}{2}+\frac{1}{2} M P_{k}\right), \quad \operatorname{Im}\left[Y_{m, n}\right] \sim N\left(0, \frac{M \sigma^{2}}{2}+\frac{1}{2} M P_{k}\right)
$$

According to (38) and (39), we can get the final distribution of $Y_{m, n}$

$$
\begin{gathered}
H_{0}: \bar{Y}_{m, n} \sim \mathrm{N}\left(0, M \sigma^{2}\right) \\
H_{1}: \bar{Y}_{m, n} \sim \mathrm{N}\left(0, M P_{k}+M \sigma^{2}\right)
\end{gathered}
$$

According to [3], the logarithmic likelihood $\operatorname{ratio} l_{m, n}$ is:

$$
l_{m, n}=\frac{P_{k} y_{m, n}^{2}}{2\left(P_{k}+\sigma^{2}\right) \sigma^{2}}+\frac{1}{2} \ln \left\{\frac{\sigma^{2}}{P_{k}+\sigma^{2}}\right\}
$$

$\forall n$, the average run length of the worst detection delay $\overline{T_{1}}$ is:

$$
\bar{T}_{1 n} \leq \frac{\gamma}{D\left(f_{1 n} \| f_{0 n}\right)}
$$

where $D\left(f_{1 n} \| f_{0 n}\right)=\frac{1}{2} \ln \left\{\frac{\sigma^{2}}{P_{k}+\sigma^{2}}\right\}+\frac{P_{k}}{2 \sigma^{2}}$. The average run length of false alarm interval $\overline{T_{0}}$ is:

$$
\bar{T}_{0 n} \geq e^{\lambda}
$$

We can get the final results on different primary user perceived performance index, and $\overline{T_{1}}$ is:

$$
\bar{T}_{1 k} \leq \frac{M \gamma}{D\left(f_{1 k} \| f_{0}\right)}
$$

$\overline{T_{0}}$ is:

$$
\bar{T}_{0} \geq M e^{\lambda}
$$

We can get the threshold $\lambda$ from (45), and can get $\overline{T_{1}}$ of different primary users by (46).

Fig. 1 A typical realization of the CUSUM and improved method statistic, when $\tau=100$

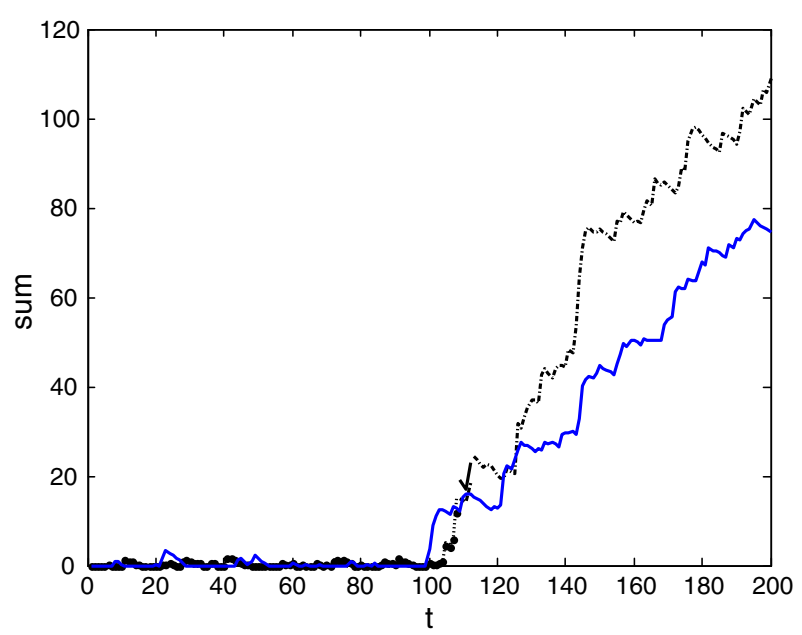




\section{Simulations and Results}

In this section, we provide some numerical results. In the simulation of method 1 , we set $\sigma^{2}=1$ and $P=3$. In the simulation of method 2, we assume that $\sigma^{2}=1$ and there are five primary users which are BPSK modulated signals with the bandwidth of $6 \mathrm{MHz}$. All the primary users have the same $P$ for simplicity. Different primary users start transmission at the 101th point, 201th point, 301th point, 401th point, and 501th point, respectively. We set the window length is 20 sampling points, and the movement of the window is nonoverlapping. In generating the simulations, the thresholds are set using the bounds for $\overline{T_{0}}$ derived in the paper.

Firstly we show some results about method 1 . Figure 1 shows a typical realization of the CUSUM and improved method statistic, when the primary user starts transmission at $\tau=100$. In the comparison, black is the new method, and blue is the CUSUM. From the

Fig. 2 Distribution of the detection delay in the CUSUM detection

Fig. 3 Distribution of the detection delay in the new method detection
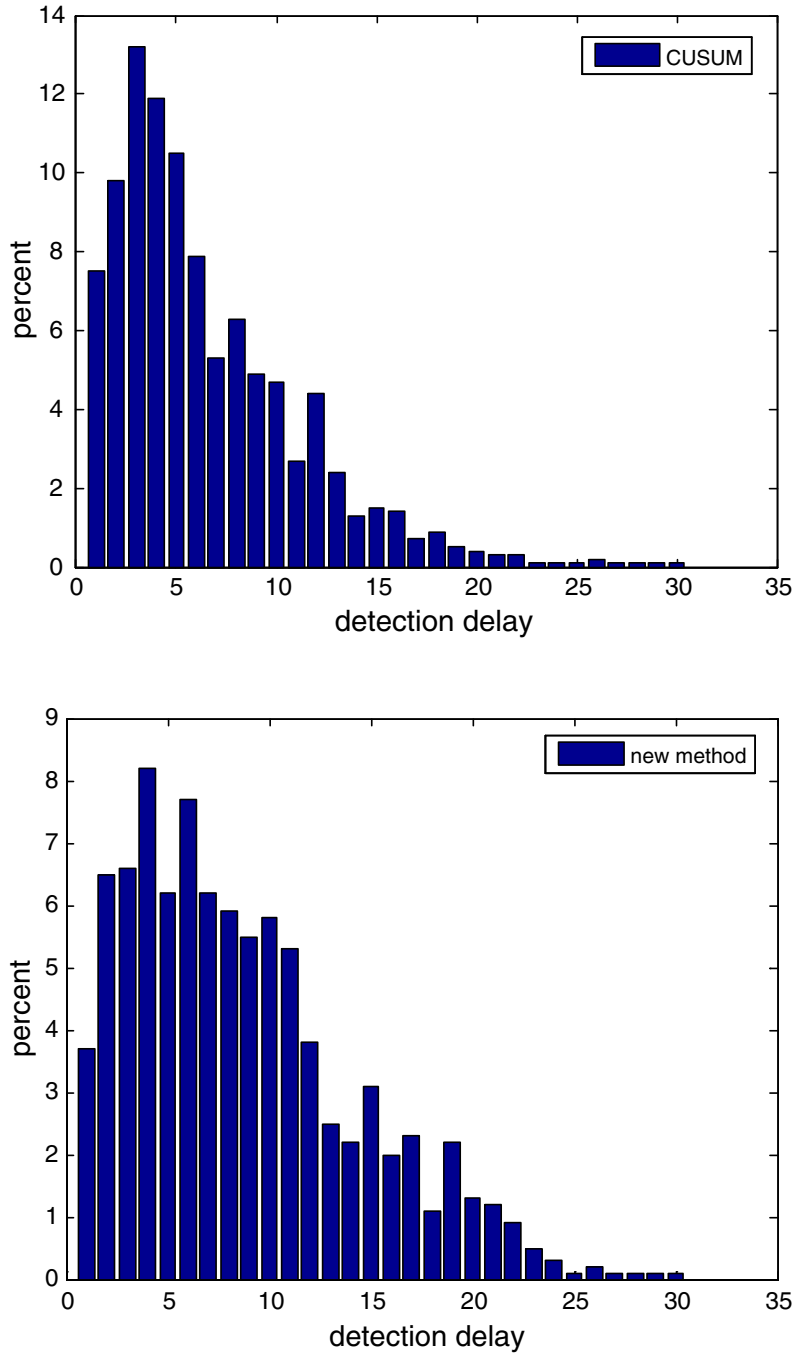
Fig. 1, we can see the cumulative sum increases rapidly of the improved method after $t=100$, thus a large difference between before the primary users appearing and after. It is very easy to detect the change of the spectrum activities with an appropriate threshold.

Figures 2 and 3 show the distributions of the detection delay in the improved method and CUSUM detection. We can see that detection delay is concentrate from 0 to 12 . When the detection delay is higher than 13 , the percent is very small in the both CUSUM and the improved method. Though we do not know the channel parameters, the new improved method still achieves the same good performance with the CUSUM detection.

Figure 4 shows the comparison between method 1 and CUSUM detection subjected to the same $\overline{T_{0}}$ when $P=3$. On the one hand, the growth rate of the detection time and the detection delay in CUSUM detection is faster than method 1. On the other hand, the high detection delay in the new method is substantially lower than CUSUM detection.

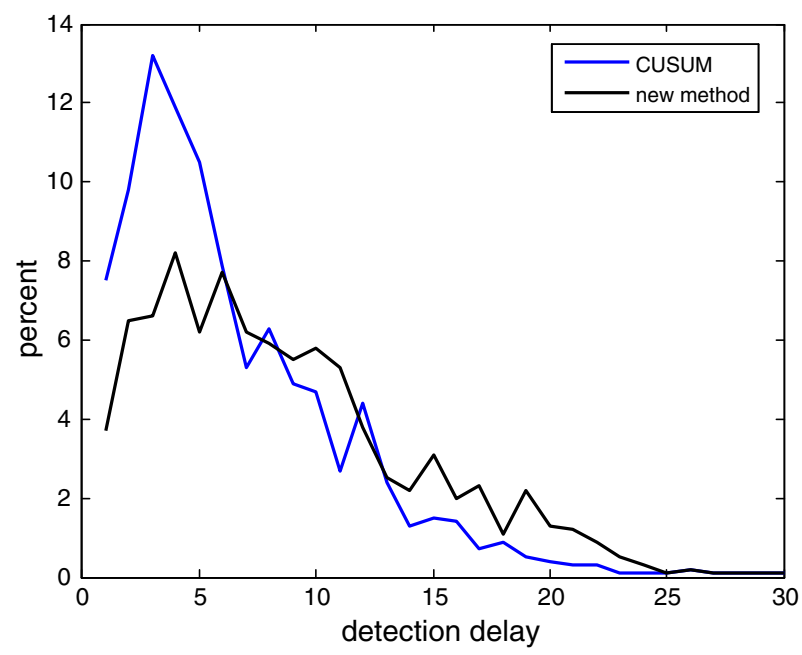

Fig. 4 Comparison of the detection delay distribution in the new method and CUSUM detection

Fig. 5 The relationship between the time and frequency of the five primary users

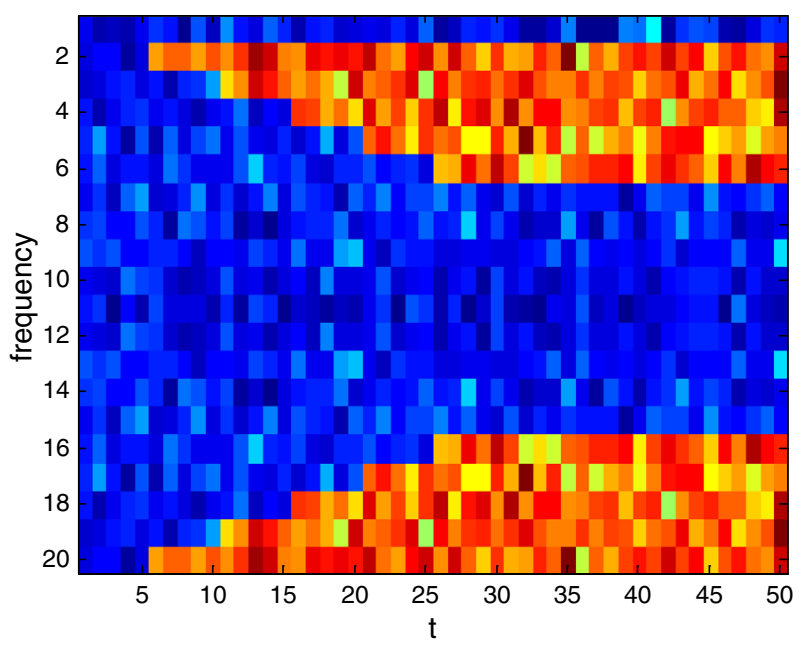


Then we also show some results about method 2. Figure 5 shows the relationship between the time and frequency of the five primary users. From the figure, we can see the time-frequency distribution after Gobar transform. The colors represent the energy, the deeper the color, the greater the energy. And by converting the time domain into frequency domain, we can clearly distinguish five primary users of different frequencies which appear at different time respectively.

Figure 6 shows the distribution of the detection delay in the wideband spectrum sensing with STFT. Figure 7 shows the distribution of the detection delay in the wideband spectrum sensing with Gobar transform. Figure 8 shows the distribution of the detection delay in the wideband spectrum sensing with Wigner-Ville transform. From the simulation results, we can see that, the delay distribution of the three kinds of transformation detection is relatively concentrated, which can be applied in practical engineer easily. Besides, the

Fig. 6 Distribution of the detection delay in the STFT
Fig. 7 Distribution of the detection delay in the Gobar
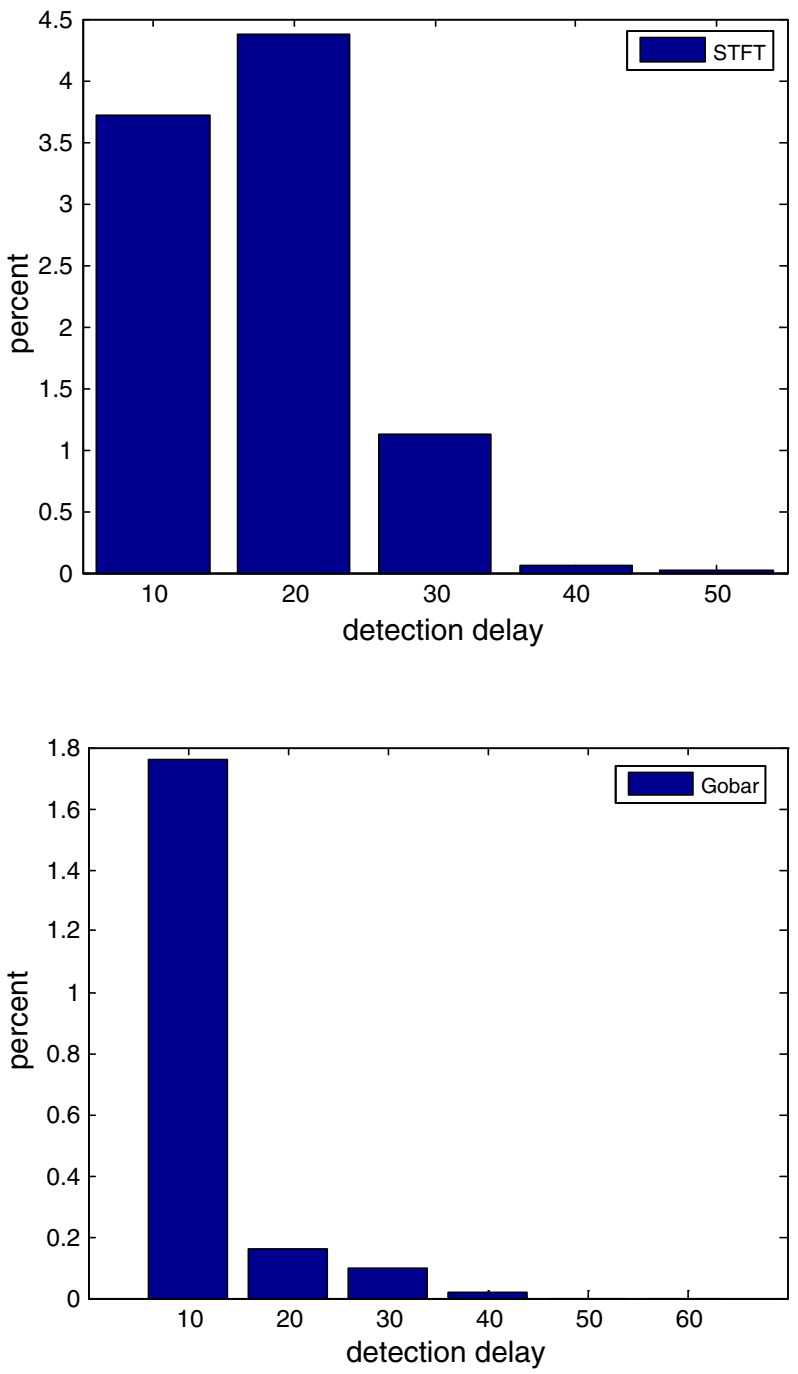
Fig. 8 Distribution of the detection delay in the WignerVille

Fig. 9 Comparison of the detection delay distribution in the Wigner-Ville, STFT and Gobar
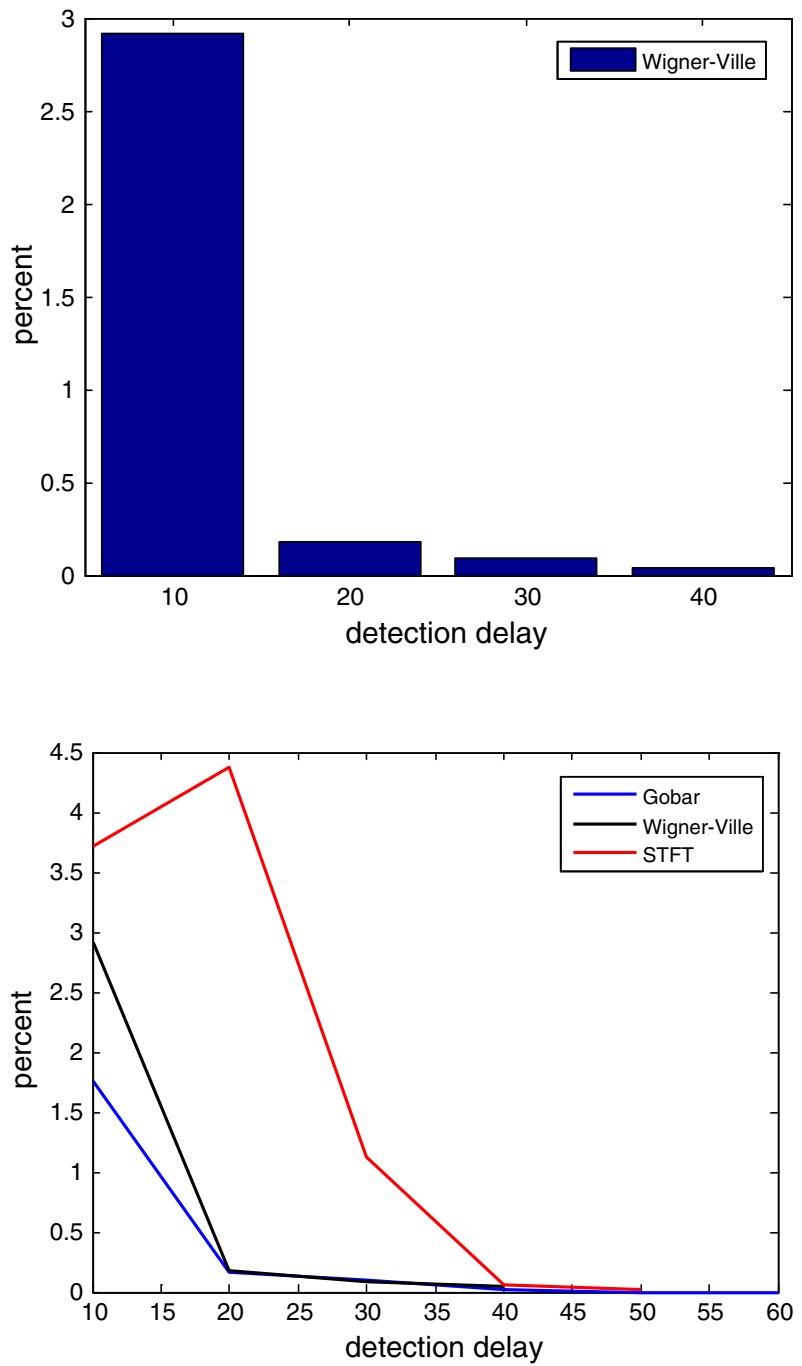

probability of detection delay of more than 20 is already very small, and compared with the signal appearing time 300 , the result is acceptable.

Figure 9 shows comparison of the detection delay distribution in the wideband spectrum sensing with Wigner-Ville, STFT and Gobar. By comparing the three kinds of transformation, we can see that the energy is relatively large and concentrated after STFT, applied to the primary user signal is close to the noise signal or even smaller than the noise signal. But STFT has a relatively large delay. Gobar and Wigner-Ville transform energy is relatively small, which can be used for the primary user signal is larger than the noise signal. On the other hand, Gobar and Wigner-Ville can easily distinguish between the various signals of different frequency, and detection delay is relatively small. Whether it is STFT, Gobar, or Wigner-Ville, detection delay is within the acceptable range. 


\section{Conclusions}

To solve the problem of spectrum sensing, this paper proposes two kinds of the quickest detection. One is applied to the signal band detection with unknown parameters of primary user. The other performs wideband spectrum sensing. In the detection, we have taken a sequential change detection framework for studying the agility of detection algorithms in cognitive radio. We focus on how to reduce the detection delay under the certain the false alarm interval, and carry out a number of experiments about the distribution of detection delay of various algorithms. Detection delay of the new method can be comparable to the CUSUM with unknown parameters of primary user in a single channel. In the wideband spectrum sensing, we continue to study the Gobar transform and Wigner-Ville transform, and carry on comparisons among them. Then we find their own suitable occasion, and previous greatly shortens the detection time, improve the detection efficiency compared with block detection. Wideband spectrum sensing will continue to focus on the unknown parameters of primary user, thus increasing spectrum efficiency.

Open Access This article is distributed under the terms of the Creative Commons Attribution 4.0 International License (http://creativecommons.org/licenses/by/4.0/), which permits unrestricted use, distribution, and reproduction in any medium, provided you give appropriate credit to the original author(s) and the source, provide a link to the Creative Commons license, and indicate if changes were made.

\section{References}

1. Poor, H. V., \& Hadjiliadis, O. (2009). Quickest detection (Vol. 40). Cambridge: Cambridge University Press.

2. Lucas, J. M., \& Crosier, R. B. (1982). Robust CUSUM: A robustness study for CUSUM quality control schemes. Communications in Statistics-Theory and Methods, 11(23), 2669-2687.

3. Li, H., Li, C., \& Dai, H. (2008). Quickest spectrum sensing in cognitive radio. In Information Sciences and Systems, 2008. CISS 2008. 42nd Annual Conference on (pp. 203-208). IEEE.

4. Perera, L. N. T., \& Herath, H. M. V. R. (2011). Review of spectrum sensing in cognitive radio. In 2011 6th international conference on industrial and information systems (pp. 7-12). IEEE.

5. Tian, Z., \& Giannakis, G. B. (2006). A wavelet approach to wideband spectrum sensing for cognitive radios. In 2006 1st international conference on cognitive radio oriented wireless networks and communications (pp. 1-5). IEEE.

6. Quan, Z., Cui, S., Sayed, A. H., \& Poor, H. V. (2008). Wideband spectrum sensing in cognitive radio networks. In 2008 IEEE international conference on communications (pp. 901-906). IEEE.

7. Lorden, G. (1971). Procedures for reacting to a change in distribution. The Annals of Mathematical Statistics, 42(6), 1897-1908.

8. Lai, L., Fan, Y., \& Poor, H. V. (2008). Quickest detection in cognitive radio: A sequential change detection framework. In IEEE GLOBECOM 2008-2008 IEEE global telecommunications conference (pp. 1-5). IEEE.

9. Zhao, Q., Li, X., \& Wu, Z. (2014). Quickest detection of multi-channel based on STFT and compressed sensing. Wireless Personal Communications, 77(3), 2183-2193.

10. Huang, J., Zhou, H., Chen, Y., Chen, B., \& Kong, R. (2014). Distributed and centralized schemes for channel sensing order setting in multi-user cognitive radio networks. Wireless Personal Communications, 75(2), 1391-1410.

11. Cabric, D., Mishra, S. M., \& Brodersen, R. W. (2004). Implementation issues in spectrum sensing for cognitive radios. In Signals, systems and computers, 2004. Conference record of the thirty-eighth Asilomar conference on (Vol. 1, pp. 772-776). IEEE.

12. Sun, H., Nallanathan, A., Wang, C. X., \& Chen, Y. (2013). Wideband spectrum sensing for cognitive radio networks: A survey. IEEE Wireless Communications, 20(2), 74-81.

13. Liang, Y. C., Zeng, Y., Peh, E. C., \& Hoang, A. T. (2008). Sensing-throughput tradeoff for cognitive radio networks. IEEE Transactions on Wireless Communications, 7(4), 1326-1337. 

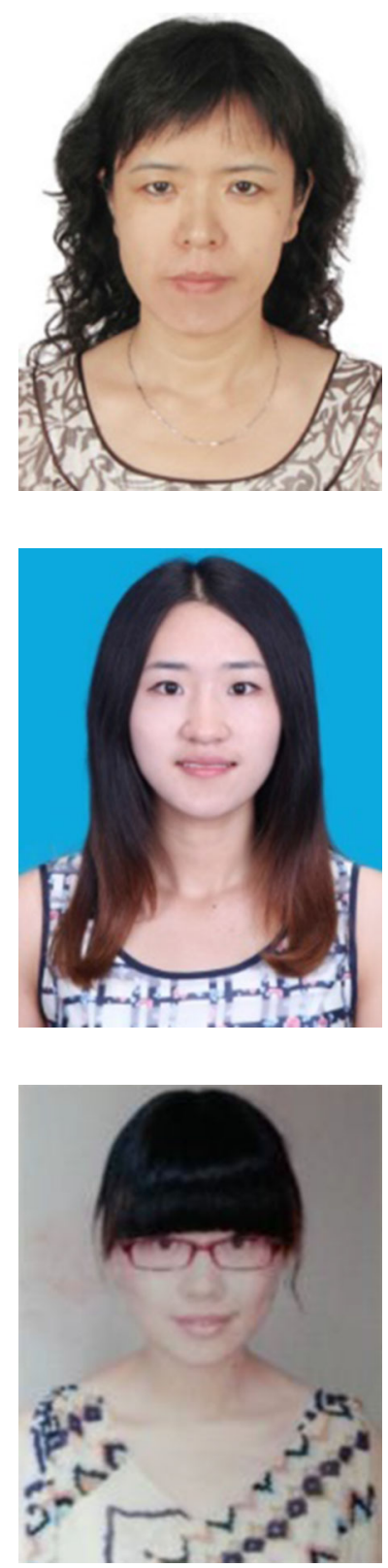

Qi Zhao received Ph.D in communication and information system from Beihang University, Beijing, China, in 2002. From 2002 to date, she is a vice-professor and work in Beihang University. Her current research interests include communication signal processing and cognitive radio technology.

Bingqian Wang received B.S. degree in electronic and information engineering from Beihang University, Beijing, China, in 2014. Now she continues to be engaged in M.S. degree study in Beihang University. Her current research interests include spectrum sensing and quickest detection.

Xiaochun $\mathbf{L i}$ received B.S. degree in electronic and information engineering from Zhengzhou University, Zhengzhou, China, in 2012. Now she is a M.S. candidate in communication and information system at Beihang. Her research interests include radio cognitive, and spectrum sensing. 